\title{
Transparency in peer review
}

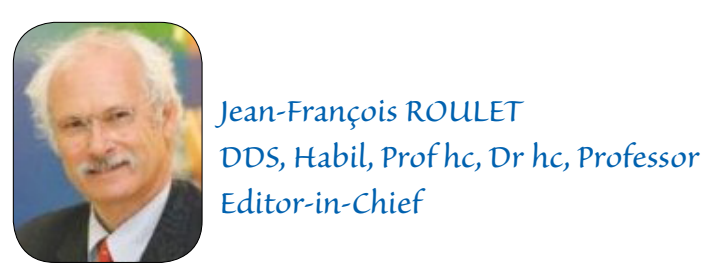

Dear readers, Dear authors,

Review is something that can have all shades of grey, even black. The black ones are allegedly reviewed, unfortunately a practice that can be observed in the literature. I can read "peer reviewed" published papers where I wonder if anybody with a functioning brain has ever read it. In the grey area, you find things as if copied from an advertisement: "Reduce the editorial time by proactively inviting the articles reviewed via our tools and reports" or "...article reports for language, plagiarism, reference accuracy \& actuality will also help you to review the articles". This sounds as if machines were doing the review.

My question is whether this software has the finesse of your trade. The next version is the editor's review. An editor may be excellent in picking up flawed language or unprecise diction. But again, no editor may be that smart and knowledgeable in order to pick scientific errors in a very specialized area of dentistry. Therefore, we rely on peer review, which means that the editor has a list of potential reviewers that are actively working on the specialty of the manuscript that needs to be reviewed. The backside of this approach is that the more qualified and specialized such a person is, the busier he/she usually is. Consequently, it has become a real pain to find good reviewers, willing to read and evaluate a manuscript in a relatively short time. In order to improve the peer review process, we decided to do a major investment and purchase the Manuscript Manager (www.manuscriptmanager.com). I can proudly announce that the Issue Stomatology Edu Journal 2019; 6 (1) is the first issue that was produced with manuscripts that were processed with the Manuscript Manager.

Once a manuscript is submitted by an author, the editor is automatically warned by an e-mail, which reduces the waiting time to be processed. The editor gets all the files in the original for the initial formal assessment. Then based on the content of the manuscript, the editor can select from a databank of specialized reviewers the best for the given manuscript. In the reviewer databank the qualification as well as the review history is visible on a click. Once selected, the potential reviewers are linked to the manuscript, while in the background the system compiles a pdf file for the reviewers. The editor can now create a priority list for the selected reviewers and invite the two most prominent ones for the review. With another click, an e-mail is created to invite the reviewer and the PDF file is automatically attached. The powerful thing about the Manuscript Manager is that it can be programmed to remind the invited reviewers if they did not answer or if they are late with submitting the review. Furthermore, the system switches automatically to the next reviewer on the list, if some time limits established for the reviewers are exceeded. By doing this we do not unnecessarily waste some time. Once two reviews are returned, again the editor gets an e-mail "decision required. If a revision is needed, which is most often the case, usually, if the authors follow the instructions to create a table with the reviewers' comments, the author' reaction and actions, the editor is then able to come up with a decision, which follows the mechanisms described above. On top of this, the system automatically keeps a log book of the events. The editor can exactly follow every step of the process and follow all e-mails exchanged as well. Thus, we get the guarantee that the peer review was done well and we also have transparency.

Summa summarum, with this outstanding tool we can serve the readers better with quality checked manuscripts and the authors with a transparent procedure. If needed, we can document the process for every single manuscript, which is important to position the Stomatology Edu Journal on a high scientific level.

Sincerely yours,

J-Froulet ${ }^{(\mathbb{P}}$

Editor-in-Chief

DOI: https://doi.org/10.25241/stomaeduj.2019.6(1).edit.3 